\title{
MODal ENergy Analysis (MODENA)
}

\author{
N. Totaro $^{1}$ and J.L. Guyader \\ Laboratoire Vibrations Acoustique - INSA-Lyon - Bâtiment St Exupery \\ 20, avenue Albert Einstein - F69621 Villeurbanne Cedex - France
}

\begin{abstract}
Energy methods like Statistical Energy Analysis (SEA) or Statistical modal Energy distribution Analysis (SmEdA), based on the well known equations of two coupled oscillators, are both limited when non-resonant contributions of modes are not negligible (typically in the case of cavity/structure/cavity coupling). In SEA, this non-resonant contribution can be taken into account introducing indirect coupling between subsystems. In SmEdA, the non-resonant contribution is more difficult to estimate as indirect coupling is not allowed. However, this issue can be a matter of importance to compute Transmission Loss (TL) of highly damped structures for example.

The present work deals with an energy method, developped within the framework of SmEdA, which solves the system of equations of two coupled oscillators at pure tone, taking thus intrinsically into account the non-resonant contributions of oscillators. As in SEA or SmEdA, the net exchanged power between two coupled oscillators is proportional to the weighted difference of total energies of oscillators. The expression of a critical coupling strength is also proposed and may be related to classical weak coupling criterion of SEA.

Extending equations obtained for two coupled sets of oscillators to the case of two linear continuous subsystems, one can compute easily frequency dependent modal energies of modes, total energies of subsystems, power transmitted between two modes and power dissipated.

The theoretical bases and assumptions of the proposed MODal ENergy Analsys (MODENA) are first exposed and the case of two coupled oscillators is addressed. Then, Plate/Cavity and Cavity/Plate/Cavity systems are treated with MODENA and compared to an exact solution. Finally, it is demonstrated that the non-resonant contribution of a highly damped plate is correctly represented by MODENA.
\end{abstract}

Keywords: Statistical Energy Analysis, SmEdA, weak coupling, non-resonant modes, Transmission Loss, MODal ENergy Analysis

\section{Introduction}

For the computation of responses of vibro-acoustic systems, two philosophies oppose. First, a deterministic approach based on the division of the domains in finite elements,

\footnotetext{
${ }^{1}$ Corresponding author. Tel.: + 33172436215. E-mail address: nicolas.totaro@insa-lyon.fr 
the so-called Finite Element Method (FEM), is a powerfull and widely used tool to predict frequency response of the whole system. FEM provides fine and almost exact results (provided that the mesh of elements is fine enough and that the characteristics of the structures are exactly known). This method is adapted for any geometry and excitation making it virtually universal. However, it suffers from some defects: (i) as the mesh size depends on frequency it is limited to low to medium frequency range, (ii) physical variability of characteristics raises the question of real meaning of computed modes, (iii) the huge quantity of information (typically millions of degrees of freedom and thousands of modes) makes the interpretation difficult. Second, a statistical approach, the so-called Statistical Energy Analysis (SEA) [1, 2, 3], estimates energy exchanges between coupled subsystems. Based on the extension of power balance between two coupled oscillators to two continuous linear subsystems, SEA provides an almost intantaneous estimation of power flow between subsystems. In addition, SEA is representative of a population of nearly identical structures and can thus give useful information on mean and variance of responses $[4,5]$. The frequency average and the use of a very simple system of equation make SEA really easy to understand. However, the assumptions underlying these equations are difficult to fulfill [6] in the case of industrial applications and the domain of validity [7] is quite difficult to estimate in real cases. In addition, to take into account non-resonant contributions of modes, it is necessary to introduce indirect couplings $[8,9]$ or to consider resonant as well as the non-resonant response as two separate subsystems [10] otherwise the energy exchanges are underestimated.

The proposed approach is within the framework of Statistical modal Energy distribution Analysis (SmEdA) [11, 12, 13] which makes a link between these two worlds, energy methods and Finite Element Method. Compared to SEA, SmEdA approach is based on modal energies rather than global energies of subsystems and withdraws the assumption of equi-partition of modal energies made by SEA opening the way to subsystems with modal behavior and local excitations. On the other hand, as SmEdA uses eigenmodes of uncoupled subsystems it can't predict the ensemble average of a population of structures but only the response of the nominal model. In addition, SmEdA only takes into account resonant contributions of modes in a frequency band and the introduction of indirect couplings is not provided. Finally, both SEA and SmEdA deal with frequency average and only provide energy of subsystems per frequency band which was voluntary to solve a few systems of equations.

The MODal ENergy Analysis (MODENA) proposed here is based on a power balance between two oscillators at pure tone contrary to SEA method where broad band excitation is considered. As in SmEdA, this power balance is extended to the case of two continuous coupled subsystems using dual modal formulation [14]. As will be demonstrated, under some classical assumptions, the net exchanged power between two coupled oscillators still depends on the total energies of oscillators and on a modal coupling loss factor at a pure tone. This approach provides the energy frequency response of each modes taking then intrinsically into account the non-resonant contribution of modes without considering indirect couplings.

The present article is organized as follows. First, the theoretical background of MODENA and its extension to multimodal susbystems will be presented. Then, two test cases will be addressed. The plate/cavity test case will demonstrates the applicability of MODENA approach comparing it to reference calculations. Then the cavity/plate/cavity test case will be used to show that MODENA is able to take into account non-resonant 
contribution of modes of a highly damped plate.

\section{Responses of two coupled oscillators}

\subsection{Transfer functions}

Let's consider two coupled oscillators as presented in Fig. 1. Each oscillator $i$ is composed by a mass $M_{i}(i=1,2)$, a spring $k_{i}$ and a damper $\lambda_{i}$. Each oscillator is excited by a random force $f_{i}(t)$. The coupling is of gyroscopic type characterized by

$$
G=\sqrt{M_{1} M_{2}} \gamma
$$

where $\gamma$ is the gyroscopic coupling coefficient.

The temporal displacements $y_{1}(t)$ and $y_{2}(t)$ of masses $M_{1}$ and $M_{2}$ must verify the two equations of motion:

$$
\ddot{y}_{1}(t)+\frac{\lambda_{1}}{M_{1}} \dot{y}_{1}(t)+\frac{k_{1}}{M_{1}} y_{1}(t)-\sqrt{\frac{M_{2}}{M_{1}}} \gamma \dot{y}_{2}(t)=\frac{f_{1}(t)}{M_{1}}
$$

and

$$
\ddot{y}_{2}(t)+\frac{\lambda_{2}}{M_{2}} \dot{y}_{2}(t)+\frac{k_{2}}{M_{2}} y_{2}(t)+\sqrt{\frac{M_{1}}{M_{2}}} \gamma \dot{y}_{1}(t)=\frac{f_{2}(t)}{M_{2}} .
$$

Considering the Fourier transform of Eqs. (2) and (3), one can obtained the system of Eq. (4) which has the form $\mathbf{A} \mathbf{Y}=\mathbf{F}$ :

$$
\left[\begin{array}{cc}
\omega_{1}^{2}-\omega^{2}+\mathrm{j} \omega \Delta_{1} & -\mathrm{j} \omega \sqrt{\frac{M_{2}}{M_{1}} \gamma} \\
\mathrm{j} \omega \sqrt{\frac{M_{1}}{M_{2}}} \gamma & \omega_{2}^{2}-\omega^{2}+\mathrm{j} \omega \Delta_{2}
\end{array}\right]\left\{\begin{array}{c}
Y_{1} \\
Y_{2}
\end{array}\right\}=\left\{\begin{array}{c}
\frac{F_{1}}{M_{1}} \\
\frac{F_{2}}{M_{2}}
\end{array}\right\},
$$

where $\omega_{i}$ is the eigen frequency and the $\Delta_{i}=\lambda_{i} / M_{i}$ is the damping bandwith of oscillator $i$.

Four transfer functions can be easily obtained solving the system of Eq. (4): $H_{11}(\omega)$, $H_{21}(\omega), H_{12}(\omega)$ and $H_{22}(\omega)$ where the transfer functions $H_{i j}(\omega)$ is the response of mass $M_{i}$ when force $F_{j}$ is applied to oscillator $j$. The expressions of these transfer functions are given by Eqs. (5) to (8):

$$
\begin{aligned}
& H_{11}(\omega)=\frac{1}{M_{1}} \frac{\omega_{2}^{2}-\omega^{2}+\mathrm{j} \omega \Delta_{2}}{\operatorname{det} \mathbf{A}}, \\
& H_{21}(\omega)=-\mathrm{j} \omega \frac{\gamma}{\sqrt{M_{1} M_{2}}} \frac{1}{\operatorname{det} \mathbf{A}}, \\
& H_{12}(\omega)=\mathrm{j} \omega \frac{\gamma}{\sqrt{M_{1} M_{2}}} \frac{1}{\operatorname{det} \mathbf{A}}, \\
& H_{22}(\omega)=\frac{1}{M_{2}} \frac{\omega_{1}^{2}-\omega^{2}+\mathrm{j} \omega \Delta_{1}}{\operatorname{det} \mathbf{A}},
\end{aligned}
$$

where $\operatorname{det} \mathbf{A}$ is the determinant of the matrix $\mathbf{A}$. The responses of the oscillators excited by forces $F_{1}$ and $F_{2}$ simultaneously are obtained by the summation of the responses to each force:

$$
Y_{1}(\omega)=H_{11}(\omega) F_{1}(\omega)+H_{12}(\omega) F_{2}(\omega)
$$

and

$$
Y_{2}(\omega)=H_{21}(\omega) F_{1}(\omega)+H_{22}(\omega) F_{2}(\omega) .
$$




\subsection{Kinetic energies of oscillators}

The kinetic energy of oscillator 1 writes:

$$
E_{k}^{1}(\omega)=\frac{1}{4} M_{1} \Re\left(\dot{Y}_{1}(\omega) \dot{Y}_{1}^{*}(\omega)\right)=\frac{1}{4} M_{1} \omega^{2} \Re\left(Y_{1}(\omega) Y_{1}^{*}(\omega)\right),
$$

where the star $(*)$ and $\Re(\bullet)$ denote respectively the complex conjugate and real part of a complex number. Introducing Eq. (9) in Eq. (11), one can express the kinetic energy of oscillator 1 as a function of transfer functions:

$$
E_{k}^{1}(\omega)=\frac{\omega^{2}}{4} M_{1} \Re\left(\left(H_{11}(\omega) F_{1}(\omega)+H_{12}(\omega) F_{2}(\omega)\right)\left(H_{11}(\omega) F_{1}(\omega)+H_{12}(\omega) F_{2}(\omega)\right)^{*}\right) .
$$

Considering two uncorrelated forces $F_{1}(\omega)$ and $F_{2}(\omega)$ (i.e $F_{1}(\omega) F_{2}^{*}(\omega)=F_{1}^{*}(\omega) F_{2}(\omega)=0$, this is the case for two harmonic forces with random phase between 0 and $2 \pi$ for example), Eq. (12) reduces to

$$
E_{k}^{1}(\omega)=\frac{1}{4} M_{1} \omega^{2}\left(\left|H_{11}(\omega)\right|^{2} S_{1}(\omega)+\left|H_{12}(\omega)\right|^{2} S_{2}(\omega)\right),
$$

where $|\bullet|$ denotes the absolute value of a number. As well, the kinetic energy of oscillator 2 writes

$$
E_{k}^{2}(\omega)=\frac{1}{4} M_{2} \omega^{2}\left(\left|H_{21}(\omega)\right|^{2} S_{1}(\omega)+\left|H_{22}(\omega)\right|^{2} S_{2}(\omega)\right),
$$

where force auto-spectra $S_{1}(\omega)=F_{1}(\omega) F_{1}^{*}(\omega)$ and $S_{2}(\omega)=F_{2}(\omega) F_{2}^{*}(\omega)$ have been introduced.

\subsection{Potential energies of oscillators}

In the same manner, the potential energy of oscillator 1 and 2 write:

$$
E_{p}^{1}(\omega)=\frac{1}{4} K_{1}\left(\left|H_{11}(\omega)\right|^{2} S_{1}(\omega)+\left|H_{12}(\omega)\right|^{2} S_{2}(\omega)\right) .
$$

and

$$
E_{p}^{2}(\omega)=\frac{1}{4} K_{2}\left(\left|H_{21}(\omega)\right|^{2} S_{1}(\omega)+\left|H_{22}(\omega)\right|^{2} S_{2}(\omega)\right) .
$$

\subsection{Total energies of oscillators}

The total energy of an oscillator is defined as the sum of kinetic and potential energies. Then total energies of oscillators 1 and 2 can be expressed as

$$
E_{t}^{1}(\omega)=E_{k}^{1}(\omega)+E_{p}^{1}(\omega)=\frac{1}{4} M_{1}\left(\omega^{2}+\omega_{1}^{2}\right)\left(\left|H_{11}(\omega)\right|^{2} S_{1}(\omega)+\left|H_{12}(\omega)\right|^{2} S_{2}(\omega)\right)
$$

and

$$
E_{t}^{2}(\omega)=E_{k}^{2}(\omega)+E_{p}^{2}(\omega)=\frac{1}{4} M_{2}\left(\omega^{2}+\omega_{2}^{2}\right)\left(\left|H_{21}(\omega)\right|^{2} S_{1}(\omega)+\left|H_{22}(\omega)\right|^{2} S_{2}(\omega)\right)
$$

It is possible using Eqs. (17) and (18) to express $S_{1}(\omega)$ and $S_{2}(\omega)$ as a function of $E_{t}^{1}(\omega)$ and $E_{t}^{2}(\omega)$ solving the following system of equations

$$
\left[\begin{array}{cc}
\left|H_{11}\right|^{2} & \left|H_{12}\right|^{2} \\
\left|H_{21}\right|^{2} & \left|H_{22}\right|^{2}
\end{array}\right]\left\{\begin{array}{c}
S_{1} \\
S_{2}
\end{array}\right\}=\left\{\begin{array}{c}
\frac{4 E_{t}^{1}}{M_{1}\left(\omega^{2}+\omega_{1}^{2}\right)} \\
\frac{4 E_{t}^{2}}{M_{2}\left(\omega^{2}+\omega_{2}^{2}\right)}
\end{array}\right\}
$$




\subsection{Net exchanged power transmitted from oscillator 1 to oscillator 2}

The net exchanged power transmitted from oscillator 1 to oscillator 2 is only due to the gyroscopic coupling

$$
\Pi_{12}(\omega)=-G\left\langle\dot{y}_{2}^{*}(t) \dot{y}_{1}(t)\right\rangle_{t}=-\frac{1}{2} G \Re\left(\dot{Y}_{2}^{*}(\omega) \dot{Y}_{1}(\omega)\right) .
$$

Introducing Eqs. (9) and (10) in Eq. (20) and considering two uncorrelated forces, one can express the net exchanged power as a function of transfer functions

$$
\Pi_{12}(\omega)=-\frac{1}{2} G \omega^{2} \Re\left(H_{21}^{*}(\omega) H_{11}(\omega) S_{1}(\omega)+H_{22}^{*}(\omega) H_{12}(\omega) S_{2}(\omega)\right) .
$$

\subsection{Relation between net exchanged power and total energies of oscillators}

Introducing expressions of $S_{1}$ and $S_{2}$ obtained with system of Eqs. (19) in Eq. (21), it yields a relation between the net exchanged power and the total energies of oscillators as follows

$$
\Pi_{12}(\omega)=\alpha_{12}(\omega) E_{t}^{1}(\omega)-\alpha_{21}(\omega) E_{t}^{2}(\omega),
$$

where $\alpha_{i j}(\omega)$ is a coupling coefficients whose expression writes

$$
\alpha_{i j}=-\frac{2 G \omega^{2}}{M_{i}\left(\omega^{2}+\omega_{i}^{2}\right)} \frac{\Re\left(H_{j i}^{*} H_{i i}\right)\left|H_{j j}\right|^{2}-\Re\left(H_{j j}^{*} H_{i j}\right)\left|H_{j i}\right|^{2}}{\left|H_{i i}\right|^{2}\left|H_{j j}\right|^{2}-\left|H_{i j}\right|^{2}\left|H_{j i}\right|^{2}}
$$

Finally, using expressions of transfer functions (Eqs. (5), (7), (6) and (8)), the expression of coupling coefficient $\alpha_{i j}$ can be simplified in

$$
\alpha_{i j}(\omega)=\frac{2 \gamma^{2}}{\left(1+\frac{\omega_{i}^{2}}{\omega^{2}}\right)} \frac{\Delta_{j} \omega^{2}\left(\left(\omega_{i}^{2}-\omega^{2}\right)^{2}+\omega^{2} \Delta_{i}^{2}\right)+\omega^{4} \gamma^{2} \Delta_{i}}{\left(\left(\omega_{i}^{2}-\omega^{2}\right)^{2}+\omega^{2} \Delta_{i}^{2}\right)\left(\left(\omega_{j}^{2}-\omega^{2}\right)^{2}+\omega^{2} \Delta_{j}^{2}\right)-\omega^{4} \gamma^{4}}
$$

It is important to underline here that Eq. (22) which expresses the relation between net exchanged power and total energies of oscillators is exact when only one force is acting (i.e. $F_{1}=0$ or $F_{2}=0$ ) or when forces $F_{1}(\omega)$ and $F_{2}(\omega)$ are uncorrelated. In the case of totally or partially correlated forces, the net exchanged power estimated by Eq. (22) is an approximation. This assumption of uncorrelated forces is also supposed in SEA or SmEdA however in this case the frequency averaging leads to the symmetry of $\alpha_{i j}(\omega)$. One can notice that the determinant of the matrix in Eq. (19) can be equal to zero. At each angular frequency, a critical coefficient $\gamma^{\text {crit }}$ can be defined. For this particular couple of angular frequency and $\gamma$, an unique solution of Eq. (19) can't be found. The expression of $\gamma^{\text {crit }}$ is given by

$$
\left|H_{11}\right|^{2}\left|H_{22}\right|^{2}=\left|H_{12}\right|^{2}\left|H_{21}\right|^{2}
$$

that is the condition for having the determinant of matrix of Eq. (19) null. Using expressions of transfer functions, the $\gamma^{\text {crit }}$ writes

$$
\gamma^{\mathrm{crit}}=\sqrt[4]{\left(\frac{\left(\omega_{1}^{2}-\omega^{2}\right)^{2}}{\omega^{2}}+\Delta_{1}^{2}\right)\left(\frac{\left(\omega_{2}^{2}-\omega^{2}\right)^{2}}{\omega^{2}}+\Delta_{2}^{2}\right)}
$$

The physical meaning of critical coefficient $\gamma^{\text {crit }}$ will be discussed in the following sections. When condition defined by Eq. (25) occurs, the coefficient $\alpha_{i j}$ and $\alpha_{j i}$ tend to infinity at the same time. However, even at this condition, the net power exchange defined in Eq. (24) tend to a finite value as will be demonstrated in the following section. 


\subsection{Power balance between coupled oscillators}

The power injected into oscillator $i$ is equal to the sum of power dissipated by oscillator $i$ and the net exchanged power between oscillators $i$ and $j$

$$
\Pi_{i}^{\mathrm{inj}}(\omega)=\Pi_{i}^{\mathrm{diss}}(\omega)+\Pi_{i j}(\omega) .
$$

In case of viscous damping and considering Eq. (22), the power balance writes

$$
\Pi_{i}^{\mathrm{inj}}(\omega)=2 \Delta_{i} E_{k}^{i}(\omega)+\alpha_{i j}(\omega) E_{t}^{i}(\omega)-\alpha_{j i}(\omega) E_{t}^{j}(\omega),
$$

Let's consider the ratio between coupling coefficients $\alpha_{i j}$ and $\alpha_{j i}$ as

$$
\Gamma=\frac{\alpha_{i j}}{\alpha_{j i}}=\frac{\left(\omega^{2}+\omega_{j}^{2}\right)}{\left(\omega^{2}+\omega_{i}^{2}\right)} \frac{\Delta_{j}\left(\left(\omega_{i}^{2}-\omega^{2}\right)^{2}+\omega^{2} \Delta_{i}^{2}\right)+\omega^{2} \gamma^{2} \Delta_{i}}{\Delta_{i}\left(\left(\omega_{j}^{2}-\omega^{2}\right)^{2}+\omega^{2} \Delta_{j}^{2}\right)+\omega^{2} \gamma^{2} \Delta_{j}} .
$$

Using the fact that $E_{k}^{i}=\frac{E_{t}^{i}}{1+\frac{\omega^{2}}{\omega_{i}^{2}}}$, one can express the energy of oscillator $i$

$$
E_{t}^{i}(\omega)=\frac{\Pi_{i}^{\mathrm{inj}}(\omega)\left(1+\frac{\tilde{\Delta}_{j}}{\alpha_{i j}}\right)+\Pi_{j}^{\mathrm{inj}}(\omega)}{\tilde{\Delta}_{i}+\tilde{\Delta}_{j} \Gamma+\frac{\tilde{\Delta}_{i} \tilde{\Delta}_{j}}{\alpha_{j i}}}
$$

where $\tilde{\Delta}_{i}=\frac{2 \omega_{i}^{2} \Delta_{i}}{\omega_{i}^{2}+\omega^{2}}$

An interesting point concerning this relation is the singularity of the coefficients $\alpha_{i j}$ and $\alpha_{j i}$ that tend to infinity when Eq. (25) applies as already noticed in the previous section. When $\alpha_{i j}$ and $\alpha_{j i}$ tend to infinity the previous expressions of energies are undeterminated. However, as the coefficient $\Gamma$ has a finite value even if $\alpha_{i j}$ and $\alpha j i$ tend to infinity, it is possible to calculate the limit of Eq. (30)

$$
\lim _{\alpha_{j i} \rightarrow \infty}\left(E_{t}^{i}(\omega)\right)=\frac{\Pi_{i}^{\mathrm{inj}}+\Pi_{j}^{\mathrm{inj}}}{\tilde{\Delta}_{i}+\tilde{\Delta}_{j} \Gamma} .
$$

It is clear that the singularity of $\alpha_{i j}$ and $\alpha_{j i}$ is not affecting the energy of the two oscillators that have finite values. It can be noticed that the damping coefficients of oscillators are controlling the energy responses.

The net power exchanged can also be calculated in the same manner

$$
\Pi_{i j}(\omega)=\frac{\Pi_{i}^{\mathrm{inj}} \tilde{\Delta}_{i}-\Pi_{j}^{\mathrm{inj}} \tilde{\Delta}_{j} \Gamma}{\tilde{\Delta}_{i}+\tilde{\Delta}_{j} \Gamma+\frac{\tilde{\Delta}_{i} \tilde{\Delta}_{j}}{\alpha_{j i}}}
$$

Again, the limit of the net exchanged power remains finite when $\alpha_{j i}$ tends to infinity

$$
\lim _{\alpha_{j i} \rightarrow \infty}\left(\Pi_{i j}(\omega)\right)=\frac{\Pi_{i}^{\mathrm{inj}} \tilde{\Delta}_{i}-\Pi_{j}^{\mathrm{inj}} \tilde{\Delta}_{j} \Gamma}{\tilde{\Delta}_{i}+\tilde{\Delta}_{j} \Gamma} .
$$

This property was not clear in Eq. (22) where $\alpha_{i j}$ and $\alpha_{j i}$ tend to infinity when Eq. (25) applies. 


\subsubsection{Influence of gyroscopic coupling strength}

It is possible from Eqs. (22) and (24) to evaluate the influence of the gyroscopic coupling strength on the coupling factor $\alpha_{i j}(\omega)$. Fig. 2 shows the map of coupling factor $\alpha_{i j}(\omega)$ as a function of gyroscopic coupling coefficient $\gamma$ and the frequency (the colormap is limited to -50 to 100 to distinguish the whole behavior of the coupling factor). A cutting plane A-A is also represented for $\gamma=500$.

It is interesting to notice that the coupling factor $\alpha_{12}(\omega)$ and $\alpha_{21}(\omega)$ are strictly positive on the whole frequency band for $\gamma<300$ in this example. Thus, the power flow is the same as in standard SEA, the bigger the energy of an oscillator the higher it transmits power to the other oscillator. For $\gamma>300$, the behavior of coupling factor changes. Indeed, around the eigen-frequencies of oscillators, the coupling factor becomes negative suddenly. Thus, the power flow is opposite compared to standard SEA, the bigger the energy of an oscillator the higher it absorbs power from the other oscillator. This is a major difference with coupling factor derived by Lyon [1] and Newland [2] and used in SmEdA [11] that is strictly positive.

This sudden transition from a strictly positive coefficient (for $\gamma<300$ in Fig. 2) to a coefficient alternatively positive or negative may be considered as a traduction of a weak coupling between oscillators. At this point, no assumption of weak coupling is made and Eqs. 22 and 24 are exact even in the case of strong coupling provided that external forces are uncorrelated. Then, this strong coupling influence can be observed in Fig.3. Indeed, when comparing the energies of oscillators for three different values of $\gamma(\gamma=10$, $\gamma=500$ and $\gamma=1000$, only the oscillator 1 is directly excited), one can remark that for low value of $\gamma$ the energy response of oscillator 1 (Fig. 3(a)) is almost not affected by the presence of the second oscillator and the net exchanged power (Fig. 3(b)) is positive but low. In that case, both eigen-frequencies of the coupled system are rather equal to those of uncoupled oscillators.

Increasing the gyroscopic coupling coefficient $\gamma$ (see Fig. 3(c) for energies and 3(d) for net exchanged power when $\gamma=500$ ) the influence of oscillator 2 on the energy response of oscillator 1 becomes observable around the uncoupled eigen-frequency of oscillator 2 where an anti-resonance appears. The two eigen-frequencies of the coupled systems are also beginning to shift relative to eigen-frequencies of uncoupled oscillators. This behavior is even more pronounced for higher values of $\gamma$ (see Fig. 3(e) and Fig. 3(f)).

Finally, one can notice that, for high value of $\gamma(\gamma=500$ or $\gamma=1000)$ and around the second eigen-frequency, the energy of oscillator 2 is higher than energy of oscillator 1 nevertheless it is directly excited. As the net exchanged power from oscillator 1 to oscillator 2 is positive as shown in Figs. 3(d) and 3(f), one can say that the power flows from the oscillator with the lowest energy to the one with the highest energy in that frequency band (around the second eigen-frequency). This remark is in contradiction with the thermic approach of the energy exchanges between oscillators that states that power flows from oscillator with the highest energy to the one with the lowest energy. The critical coefficient $\gamma^{\text {crit }}$ defines the limit between a "thermodynamic" and an "antithermodynamic" behavior. In the example presented in Fig. 4, the value of $\gamma=500$ is plotted on the evolution of $\gamma^{\text {crit }}$ coefficient. In that case, the power flow between oscillators is almost always comparable to a "thermodynamic" behavior except near the eigen-frequencies of coupled oscillators. In that frequency bands, the coupling is too strong and an "anti-thermodynamic" behavior appears. 


\subsubsection{Error due to correlated forces}

When external forces $f_{1}(t)$ and $f_{2}(t)$ are correlated, Eqs. (22) and (24) are not exact any more because they neglect cross-spectrum between forces. It is nevertheless interesting to evaluate the error due to the use of Eq. (24) even though the forces are correlated. Fig. 5 shows the net exchanged power transmitted from oscillator 1 to oscillator 2 as a function of frequency for four different amplitude ratios of correlated forces $\left(\left|F_{1}\right| /\left|F_{2}\right|=10^{1}, 10^{2}, 10^{3}\right.$ and $\left.10^{4}\right)$. It is clear in Fig. 5 that two correlated forces, even with a high amplitude ratio, lead to high error on net exchanged power mainly far from eigen-frequencies, that is to say when the modes have low responses. Obviously, for extremely high amplitude ratio (see Fig. 5(d)) this error becomes negligible on the whole frequency range. This tends to demonstrates that Eq. (22) can be a good approximation in case of weak coupling between oscillators.

Let's assume now that at angular frequency $\omega$ the phase between both force Fourier transforms is random and that we are interested in the expectation of the responses of oscillators to this ensemble of correlated forces with random phase $(\Phi=0 . .2 \pi)$. Fig. 6 presents the expectation of net exchanged power (averaged on 2500 realizations) compared to Eq. (24) calculation. The inserted figures represent the net exchanged power computed for each of the 2500 realizations compared to the statistical average. Comparing Fig. 5 and Fig. 6, it is very clear that the error due to the use of correlated forces drastically decreases when comparing MODENA approach (which supposes uncorrelated forces) to expectation of responses of oscillators to forces with random phase. Thus, even for low amplitude ratio of forces, the error becomes negligible on the whole frequency band. This result can be associated to the ensemble average assumption in standard SEA.

\subsection{Extension to multi-modal coupling}

Let's consider two sets of modes $N_{p}$ and $N_{q}$ representing two coupled continuous subsystems as presented in Fig. 7. Using a dual modal formulation [14], as done in SmEdA approach $[11,12,13]$, a power balance for each mode of a set of modes coupled to another set of modes can be written. Modes of the uncoupled subsystems are expressed either in terms of displacement (free at the coupling interface) or in terms of stress (blocked at the coupling interface). In that case, there is no coupling between two modes of the same subsystem and the coupling between one mode of each subsystem is gyroscopic. It has been demonstrated that the modes of subsystems can be considered as sets of oscillators and that the power injected into mode $p, \Pi_{p}^{\text {inj }}(\omega)$, is either dissipated by the modal damping loss factor or transmitted to modes $q$ of a set of modes $N_{q}$

$$
\Pi_{p}^{\mathrm{inj}}(\omega)=\Pi_{p}^{\mathrm{diss}}(\omega)+\sum_{q} \alpha_{p q}(\omega) E_{t}^{p}(\omega)-\sum_{q} \alpha_{q p}(\omega) E_{t}^{q}(\omega) .
$$

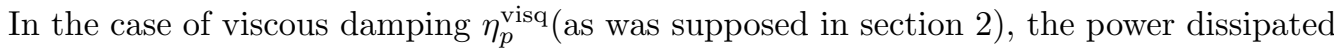
by a mode is proportional to its kinetic energy,

$$
\Pi_{p}^{\text {diss }}(\omega)=2 \eta_{p}^{\mathrm{visq}} \omega_{p} E_{k}^{p}(\omega)=2 \eta_{p}^{\mathrm{visq}} \omega_{p} \frac{E_{t}^{p}(\omega)}{1+\frac{\omega^{2}}{\omega_{p}^{2}}}
$$


whereas, in the case of structural damping $\eta_{p}^{\text {struc }}$, the power dissipated by a mode is proportional to its potential energy,

$$
\Pi_{p}^{\text {diss }}(\omega)=2 \eta_{p}^{\mathrm{struc}} \omega E_{k}^{p}(\omega)=2 \eta_{p}^{\mathrm{struc}} \omega \frac{E_{t}^{p}(\omega)}{1+\frac{\omega_{p}^{2}}{\omega^{2}}} .
$$

In addition, in the case of viscous damping, the coupling factor $\alpha_{p q}(\omega)$ writes

$$
\alpha_{p q}(\omega)=\frac{2 \gamma_{p q}^{2}}{\left(1+\frac{\omega_{p}^{2}}{\omega^{2}}\right)} \frac{\Delta_{q}^{\mathrm{visc}} \omega^{2}\left(\left(\omega_{p}^{2}-\omega^{2}\right)^{2}+\omega^{2}\left(\Delta_{p}^{\mathrm{visc}}\right)^{2}\right)+\omega^{4} \gamma_{p q}^{2} \Delta_{p}^{\mathrm{visc}}}{\left(\left(\omega_{p}^{2}-\omega^{2}\right)^{2}+\omega^{2}\left(\Delta_{p}^{\mathrm{visc}}\right)^{2}\right)\left(\left(\omega_{q}^{2}-\omega^{2}\right)^{2}+\omega^{2}\left(\Delta_{q}^{\mathrm{visc}}\right)^{2}\right)-\omega^{4} \gamma_{p q}^{4}} .
$$

whereas in the case of structural damping, this expression changes in

$$
\alpha_{p q}(\omega)=\frac{2 \gamma_{p q}^{2}}{\left(1+\frac{\omega_{p}^{2}}{\omega^{2}}\right)} \frac{\Delta_{q}^{\text {struc }} \omega_{q} \omega\left(\left(\omega_{p}^{2}-\omega^{2}\right)^{2}+\omega_{p}^{2}\left(\Delta_{p}^{\text {struc }}\right)^{2}\right)+\omega_{p} \omega^{3} \gamma_{p q}^{2} \Delta_{p}^{\text {struc }}}{\left(\left(\omega_{p}^{2}-\omega^{2}\right)^{2}+\omega_{p}^{2}\left(\Delta_{p}^{\text {struc }}\right)^{2}\right)\left(\left(\omega_{q}^{2}-\omega^{2}\right)^{2}+\omega_{q}^{2}\left(\Delta_{q}^{\text {struc }}\right)^{2}\right)-\omega^{4} \gamma_{p q}^{4}},
$$

where $\Delta_{p}^{\text {visc }}=\eta_{p}^{\text {visc }} \omega_{p}$ and $\Delta_{p}^{\text {struc }}=\eta_{p}^{\text {struc }} \omega_{p}$. The gyroscopic coefficient $\gamma_{p q}$ is proportional to the modalwork between a mode $p$ and mode $q$, expressed here in the case of a Plate/Cavity coupling [12]

$$
\gamma_{p q}=\frac{1}{\sqrt{M_{p} M_{q}}} \int_{S_{c}} \Phi_{p}^{\text {plate }}(Q) \Psi_{q}^{\text {cav }}(Q) d Q,
$$

where $S_{c}$ is the coupling surface between the plate and the cavity, $\Phi_{p}^{\text {plate }}(Q)$ is the displacement mode shape of the plate, $\Psi_{q}^{\text {cav }}(Q)$ is the pressure mode shape of the cavity and $M_{p}$ and $M_{q}$ are the modal masses.

To apply this power balance equation of two sets of oscillators, some assumptions have to be fulfilled:

(i) the coupling between two modes has to be conservative.

(ii) External forces applied on subsystems have to be uncorrelated.

(iii) Isolating two modes, the ensemble of coupling forces due to other modes of the subsystems are considered as uncorrelated to external excitations.

As already shown in sections 2.7.1 and 2.7.2, the assumption (iii) may be satisfied if the coupling is weak between both subsystems and/or if the coupling forces can be considered as forces with randomly distributed phase.

\section{Plate/Cavity coupling}

Fig. 8 presents the first test case: a plate excited by a point force coupled to a cavity with rigid walls. Properties of the cavity and the plate are listed in Tables 1 and 2. To solve this problem with the MODENA approach, the powers injected into each mode of 
the plate at angular frequency $\omega$ have to be determined. The modal power due to a point force $\mathrm{F}$ is given by

$$
\Pi_{p}^{\mathrm{inj}}(\omega)=\frac{1}{2} \Re\left(F(\omega) V_{p}^{*}(\omega)\right) .
$$

Assuming weak coupling, that is to say that modal input powers due to an unit force located at $\left(X_{e}, Y_{e}\right)=(0.7,0.2)$ can be calculated neglecting the cavity, we found

$$
\Pi_{p}^{\mathrm{inj}}(\omega)=\frac{\Delta_{p} \omega^{2}\left(\Phi_{p}\left(X_{e}, Y_{e}\right)\right)^{2}}{2 M_{p}\left[\left(\omega_{p}^{2}-\omega^{2}\right)^{2}+\left(\Delta_{p} \omega\right)^{2}\right]},
$$

where $\Phi_{p}\left(X_{e}, Y_{e}\right)$ is the mode shape of the mode $p$ at point force location.

Solving a system of $N^{\text {plate }}+N^{\text {cav }}$ equations like Eq. (34) we get the modal energies of each subsystem as a function of frequency. The coupling coefficient $\alpha_{p q}$ can be associated to viscous damping using Eq. (37) or structural damping (Eq. (38)) depending on the case of interest. Modal energies of the plate and the cavity are plotted in Fig. 9. One can first notice that, for the plate only few modes are contributing to the response at each frequency. Two groups of modes can be seen, one is controlling the response, the second has low contribution whatever the frequency because of the excitation located near their nodal lines. In the cavity the number of modes is larger and the splitting in two groups cannot be done however at each frequency the number of modes contributing significantly to the energy level is small again.

One can also notice that the frequency response of each modal energy does not follow exactly the one of an isolated oscillator and coupling effects to the modes of the other subsystem can be observed. In the case of the plate, each modal energy is influenced by modes of the cavity and blocking effects can be seen at these particular resonance frequencies of the cavity. The same remarks can be done for the modal energies of the cavity.

In the case of a rain-on-the-roof excitation (here introduced by 100 uncorrelated randomly distributed point forces), all the plate modes are equally excited and the response is no more separated in two groups of modes as shown in Fig. 10. With a rain-on-the-roof excitation, modal energies are globally identical on the frequency band corresponding to the equipartition of energy assumed in SEA energy, however when looking to the plate modal energies at a given frequency a very small number of modes are controlling the response. In Fig. 10, cavity modal energies are presented. Cavity modes spatially coupled with plate modes dominate and equipartition of energy is not achieved. In addition, at a given frequency, the number of modes participating to the energy level is small.

Fig. 11 shows the global energies (i.e the sum of each modal energy) of the plate and the cavity compared to the CVALOR reference calculation (a vibro-acoustic benchmark for simple structures [15]) and to SmEdA calculation. As can be seen, the global energy of each subsystem prediction with MODENA agrees well with reference calculations. This simple test case validates the MODENA approach to compute modal and global energies of subsystems as a function of frequency.

\section{Cavity/Plate/Cavity coupling}

Fig. 12 presents the second test case: a plate inserted in between two cavities A and B. The dimensions and properties of the cavities are presented in Table 4 and those of 
plate 1 are listed in Table 3 . The excitation is created by a single monopole in cavity A at $\left(X_{e}, Y_{e}, Z_{e}\right)=(0.1,0.1,0.1)$. The simulation is compared to a direct frequency response computed by Actran software between 50 and $700 \mathrm{~Hz}$. The MODENA calculation agrees well to this reference calculation. This result is important because standard SEA has difficulty to take into account the non-resonant contribution of plate modes and indirect coupling must be introduced for agreeing reality.

As demonstrated in this reference test case, MODENA approach is able to represent Cavity/Plate/Cavity systems for which non resonant contribution is important. Using plate 2LD (Low Damping) and plate 2HD (HighDamping) described in Table 3, one can illustrate some well known effects of damping on Transmission Loss [16]. Below the critical frequency, an increase of damping of the structure has little influence on transmission because it is dominated by non-resonant mode contributions. On the contrary, above the critical frequency, an increase of damping is efficient to improve acoustic insulation of the panel, as shown in Fig. 14.

\section{Conclusion}

The proposed approach is an energy based method but contrary to SEA or SmEdA method it provides a pure tone analysis of power flow. It has been demonstrated that the net exchanged power transmitted from one oscillator to the other only depends on total energies of oscillators and of a "modal coupling loss factor at pure tone" whose expression is a function of resonance frequencies and damping of uncoupled oscillators. The main particularity of the power flow/energy relation is the possibility of having negative coefficient contrary to SEA relation. In this case the power flows from the oscillator of low energy to that of high energy and not from the oscillator of high energy to that of low energy, this possibility is however restricted to sufficiently strong coupling.

The coupling between oscillators has been extended to the coupling between two continuous subsystems as done in SmEdA using dual modal formulation. The plate/cavity test case has been treated as example. The energies of the plate (directly excited by a point force) and the cavity have been compared to a reference calculation showing a good agreement. Then, sound transmission through a panel separating two cavites has been studied. The contribution of non-resonant plate mode to the transmission is well known in this problem and necesssitate in standard SEA the introduction of indirect coupling. In the present approach non-resonant contribution of modes is included and as expected the prediction is good.

Finally, as this approach is close to Statistical modal Energy distribution Analysis (SmEdA), the same advantages and extensions apply. Indeed, (i) the modal coupling loss factor at pure tone only depends on quantities which can be easilly obtained with finite element methods, (ii) finite element computations are only necessary on uncoupled subsystems, (iii) subsystems with low modal overlap and local excitations can be addressed and (iv) using modal energy of subsystems it is possible to estimate the energy distribution into subsystems. 


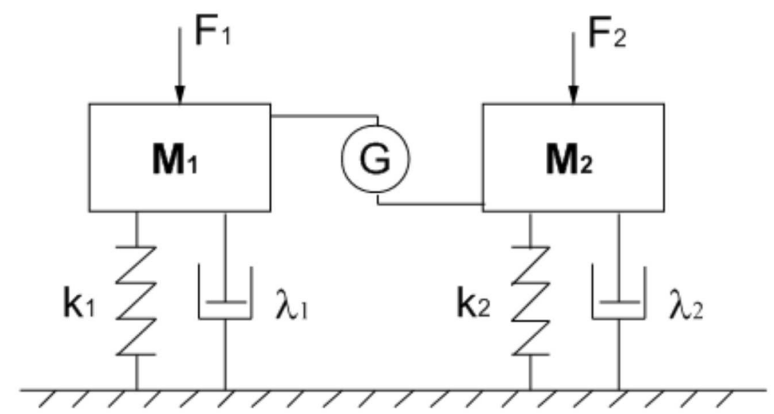

Figure 1: Sketch of two oscillators coupled by a gyroscopic coupling 


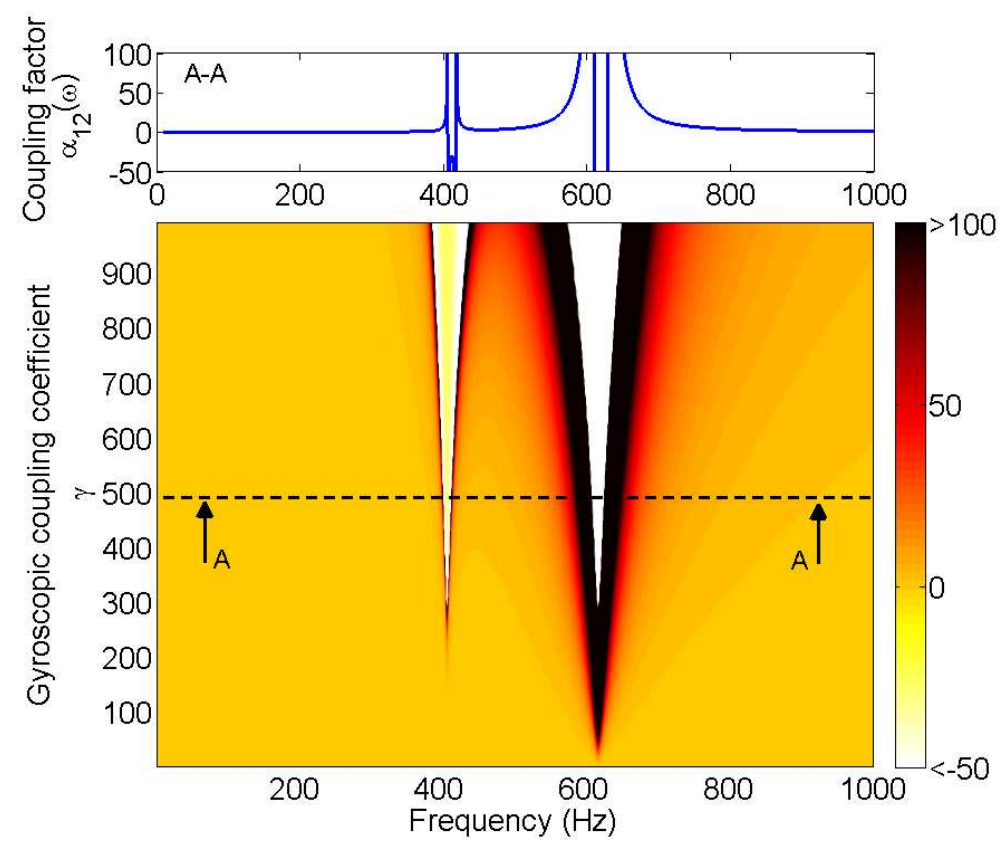

Figure 2: Coupling factor $\alpha_{12}$ as a function of frequency and of gyroscopic coupling coefficient. Top figure represents a cut of this map for $\gamma=500$. 

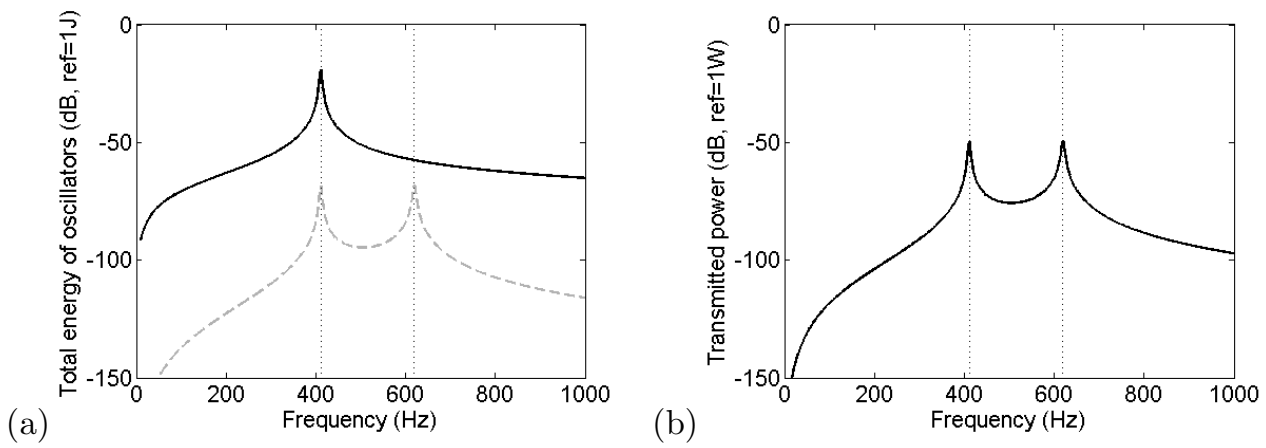

(a)

(b)

(c)

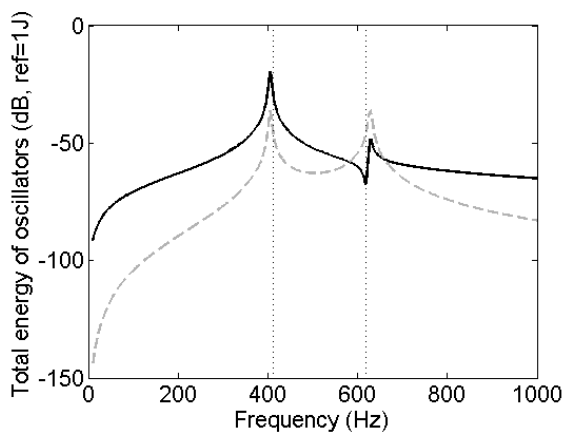

(d)
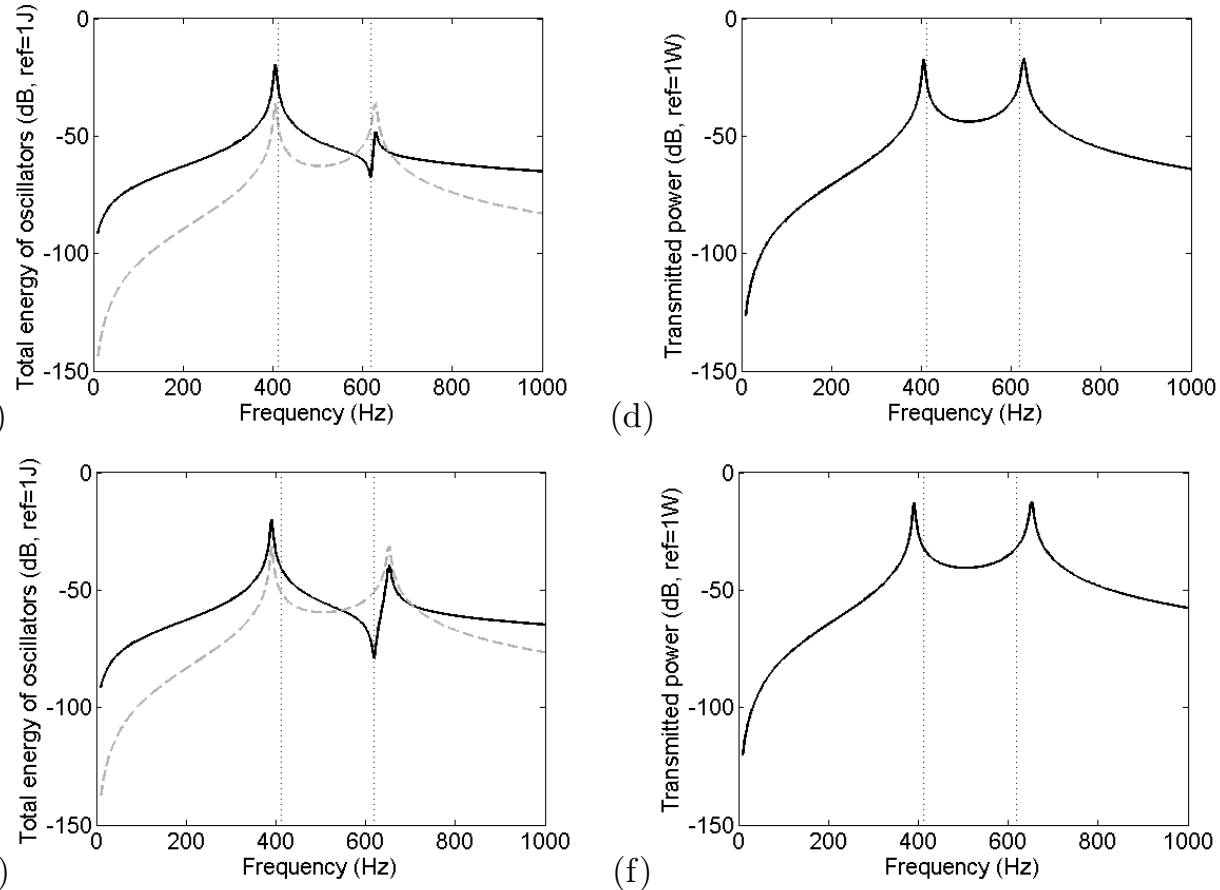

Figure 3: Total energies of oscillators 1 (solid black line) and 2 (dashed gray line) when oscillator 1 is excited by a unit force for (a) $\gamma=10$, (c) $\gamma=500$ and (e) $\gamma=1000$ and corresponding transmitted power from oscillator 1 to oscillator 2 for (b) $\gamma=10$, (d) $\gamma=500$ and (f) $\gamma=1000$. Vertical dotted lines represent the positions of eigen-frequencies of uncoupled oscillators. 


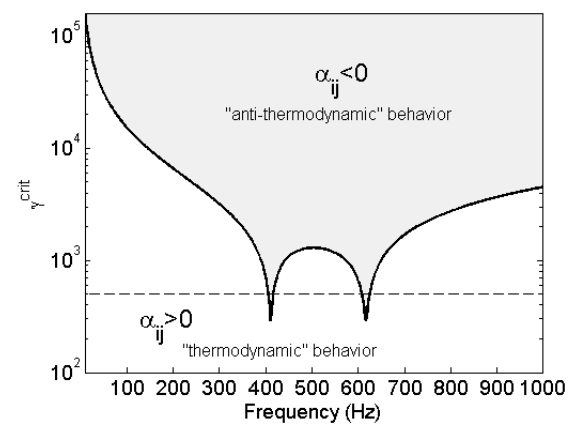

Figure 4: Critical coefficient $\gamma^{\text {crit }}$ as a function of frequency. The dashed line represents $\gamma=500$. The critical coefficient defines the limit between a "thermodynamic" behavior $\left(\alpha_{i j}>0\right)$ and an "antithermodynamic" behavior $\left(\alpha_{i j}<0\right)$.

(a)

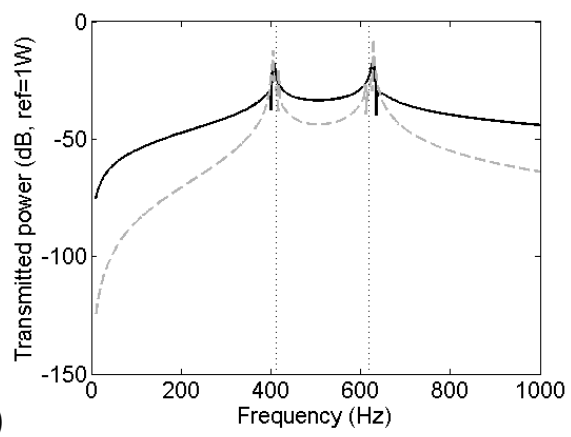

(c)

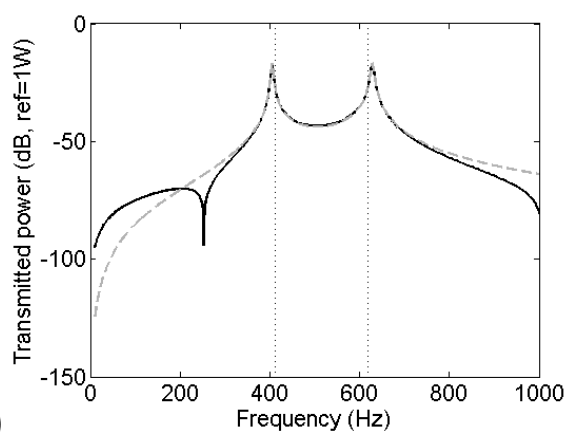

(b)

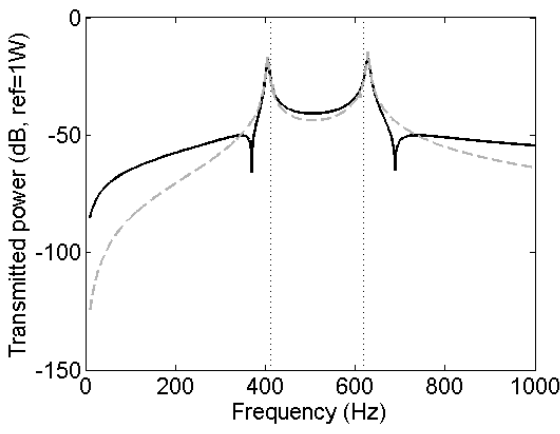

(d)

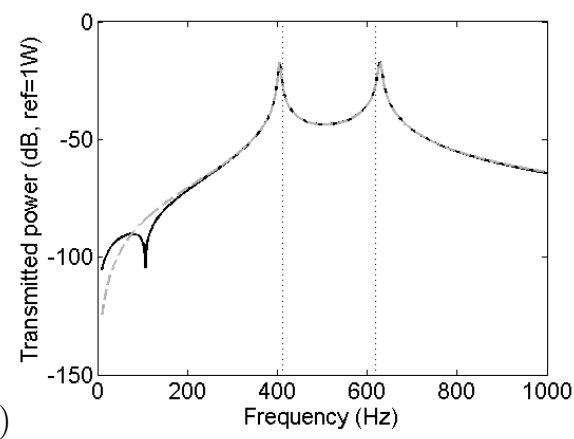

Figure 5: Power transmitted from oscillator 1 to oscillator 2 when oscillators 1 and 2 are excited by two correlated forces with (a) $\left|F_{1}\right| /\left|F_{2}\right|=10^{1}$, (b) $\left|F_{1}\right| /\left|F_{2}\right|=10^{2}$, (c) $\left|F_{1}\right| /\left|F_{2}\right|=10^{3}$ and (d) $\left|F_{1}\right| /\left|F_{2}\right|=$ $10^{4}$. Black solid line: exact analytical solution, gray dashed line: MODENA approach. Vertical dotted lines represent the positions of eigen-frequencies of uncoupled oscillators. 
(a)

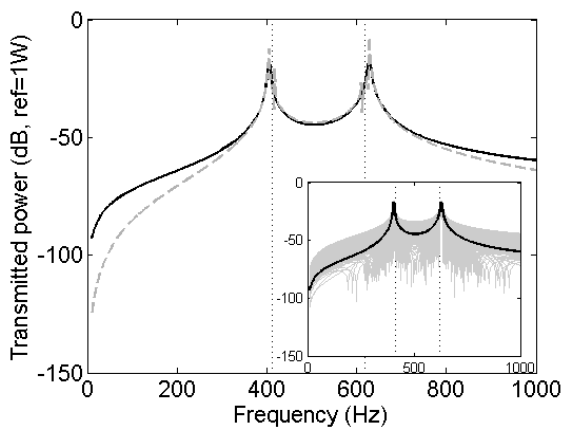

(c)

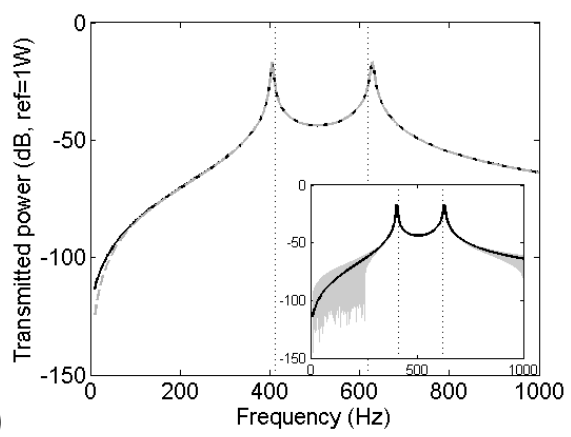

(b)

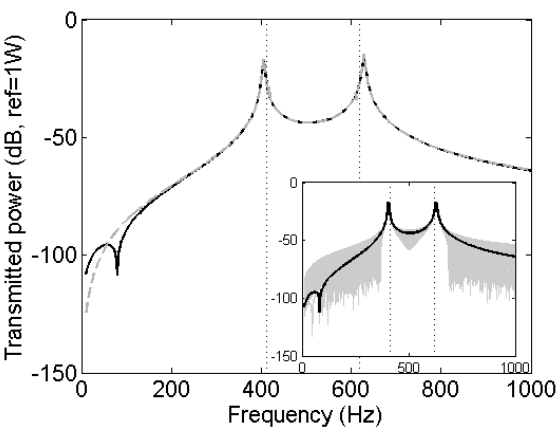

(d)

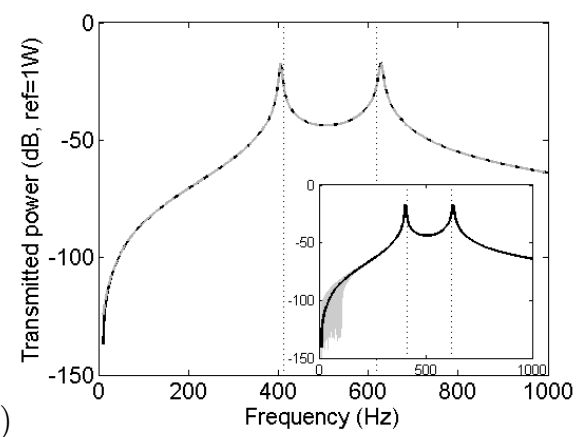

Figure 6: Power transmitted from oscillator 1 to oscillator 2 when oscillators 1 and 2 are excited by two forces with random phase with (a) $\left|F_{1}\right| /\left|F_{2}\right|=10^{1}$, (b) $\left|F_{1}\right| /\left|F_{2}\right|=10^{2}$, (c) $\left|F_{1}\right| /\left|F_{2}\right|=10^{3}$ and (d) $\left|F_{1}\right| /\left|F_{2}\right|=10^{4}$. Black solid line: averaged analytical solution over 2500 realizations with random phase from 0 to $2 \pi$; gray dashed line: MODENA approach with two correlated forces. Vertical dotted lines represent the positions of eigen-frequencies of uncoupled oscillators. The inserted figures show each of 2500 realizations with random phase (in gray) compared to the average response (in black).

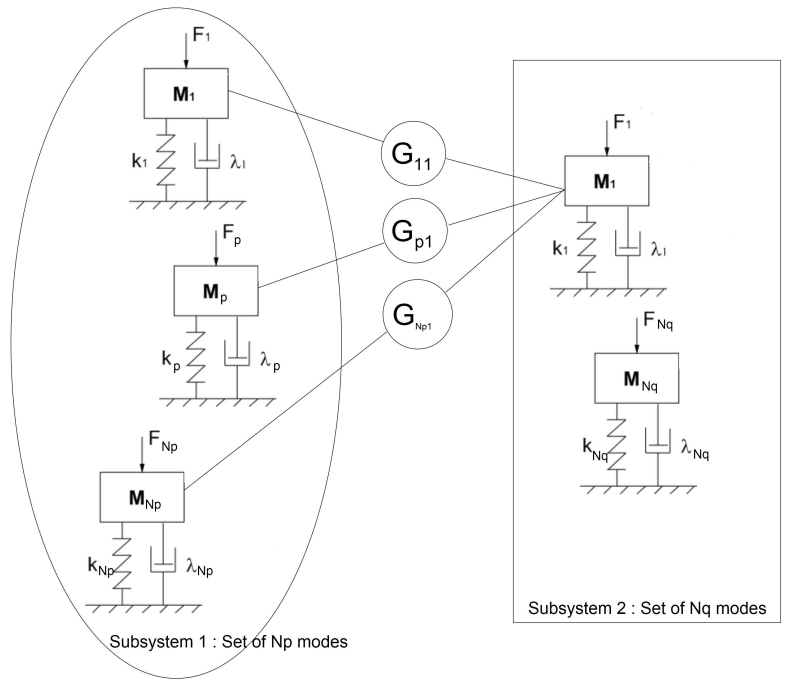

Figure 7: Coupling between two sets of modes 


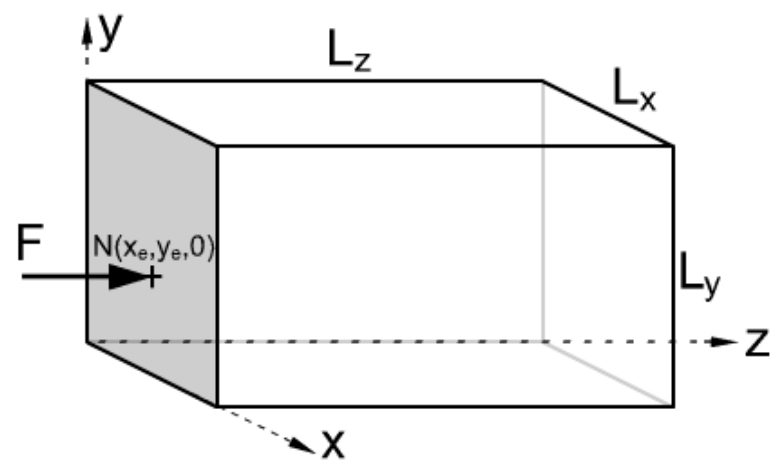

Figure 8: Sketch of Plate/Cavity coupling test case. The plate is excited by a point force at $\left(x_{e}, y_{e}\right)$

(a)

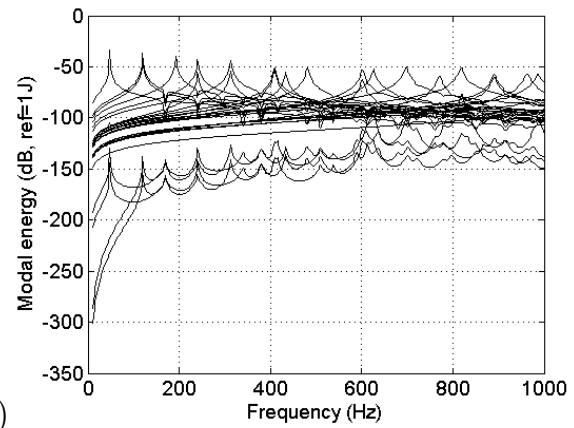

Figure 9: Modal energies of modes of (a) the plate and (b) the cavity when the plate is excited by a single point force.

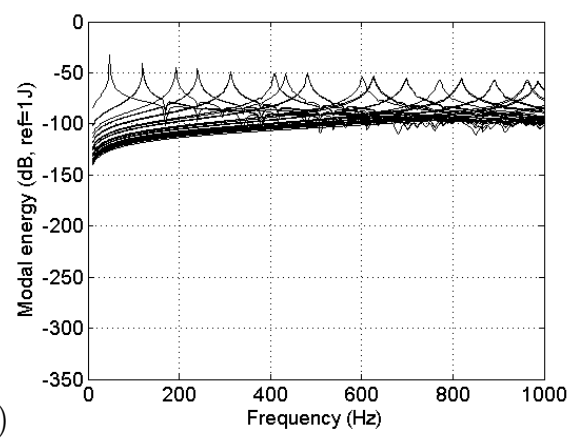

(b)

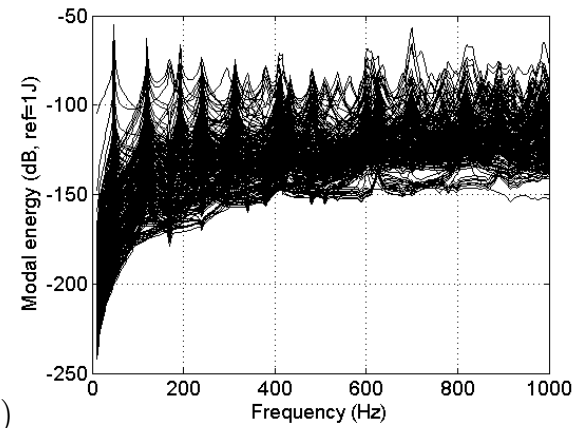

(b)

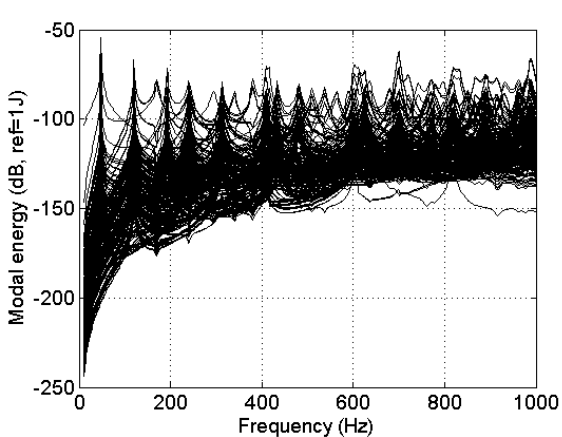

Figure 10: Modal energies of modes of (a) the plate and (b) the cavity when the plate is excited by a rain-on-the-roof excitation (100 uncorrelated point forces of amplitude $1 / 100 \mathrm{~N}$ ). 

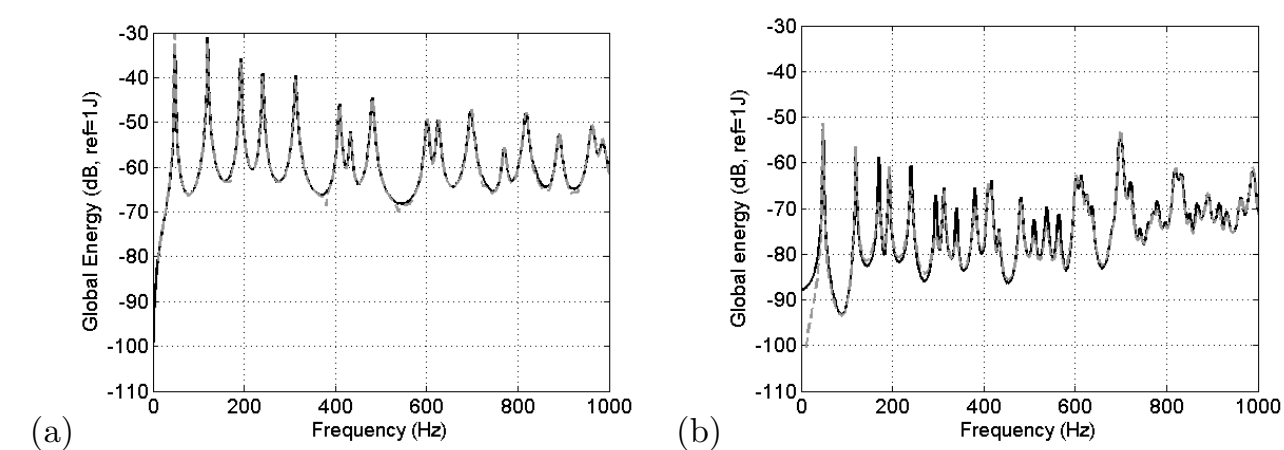

Figure 11: Total energy of (a) the plate and (b) the cavity as a function of frequency. Solid black line : CVALOR reference calculation; dashed gray line : MODENA calculation. The plate is excited by one point force.

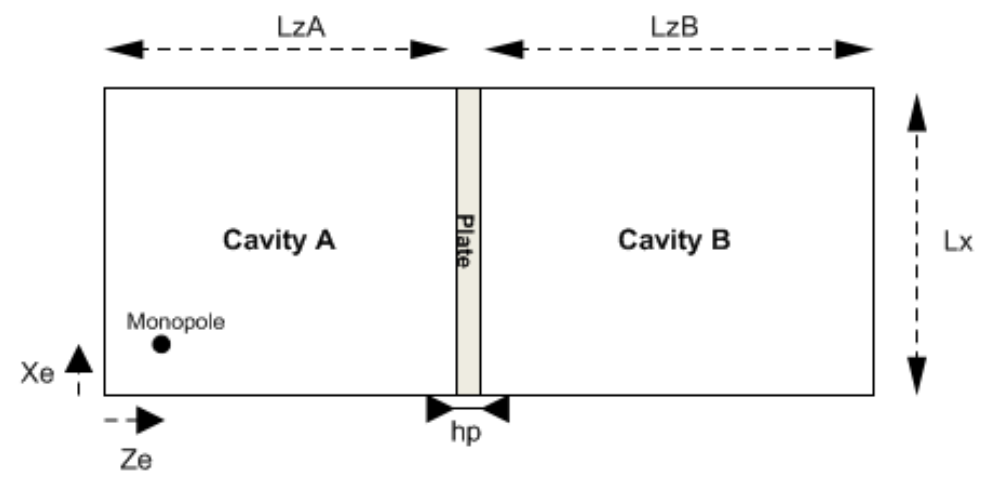

Figure 12: Sketch of Cavity/Plate/Cavity coupling test case. The cavity is excited by a monopole at $\left(X_{e}, Y_{e}, Z_{e}\right)$

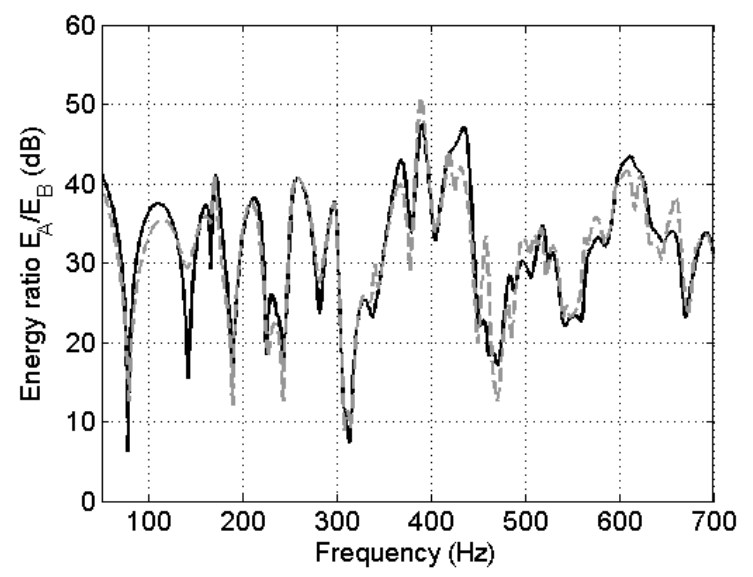

Figure 13: Energy ratio between cavity A and cavity B when cavity A is excited by one monopole. Black solid line : ACTRAN reference calculation; Dashed gray line : MODENA calculation. 


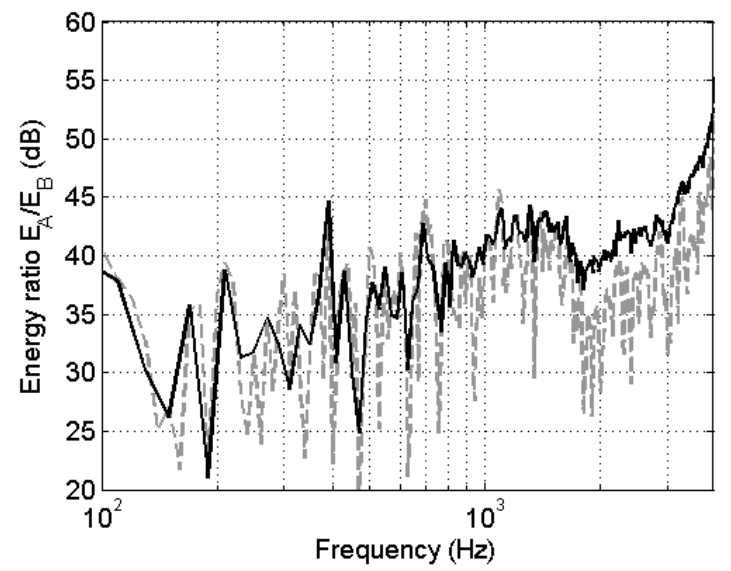

Figure 14: Energy ratio between cavity A and cavity B when cavity A is excited by 20 randomly located monopoles. Solid gray line : MODENA calculation (Plate 2LD, damping 1\%); Solid black line : MODENA calculation (Plate 2HD, damping 5\%). 


\begin{tabular}{|c|c|}
\hline & Plate \\
\hline$L_{x}(\mathrm{~m})$ & 1 \\
\hline$L_{y}(\mathrm{~m})$ & 1 \\
\hline$E_{p}(\mathrm{~Pa})$ & $2 \mathrm{e} 11$ \\
\hline$\rho_{p}\left(\mathrm{~kg} / \mathrm{m}^{3}\right)$ & 7800 \\
\hline$\nu_{p}$ & 0.3 \\
\hline$h_{p}(\mathrm{~mm})$ & 10 \\
\hline$\eta_{p}^{\text {visc }}$ & 0.01 \\
\hline
\end{tabular}

Table 1: Characteristics of the plate for the Plate/Cavity test case

\begin{tabular}{|c|c|}
\hline & Cavity \\
\hline$L_{x}(\mathrm{~m})$ & 1 \\
\hline$L_{y}(\mathrm{~m})$ & 1 \\
\hline$L_{z}(\mathrm{~m})$ & 1 \\
\hline$\rho_{c}\left(\mathrm{~kg} / \mathrm{m}^{3}\right)$ & 1.2 \\
\hline$c_{c}(\mathrm{~m} / \mathrm{s})$ & 340 \\
\hline$\eta_{c}^{\text {struc }}$ & 0.01 \\
\hline
\end{tabular}

Table 2: Characteristics of the cavity for the Plate/Cavity test case

\begin{tabular}{|c|c|c|c|}
\hline & Plate 1 & Plate 2LD & Plate 2HD \\
\hline$L_{x}(\mathrm{~m})$ & \multicolumn{3}{|c|}{0.9} \\
\hline$L_{y}(\mathrm{~m})$ & \multicolumn{3}{|c|}{$2 \mathrm{e} 11$} \\
\hline$E_{p}(\mathrm{~Pa})$ & \multicolumn{3}{|c|}{7800} \\
\hline$\rho_{p}\left(\mathrm{~kg} / \mathrm{m}^{3}\right)$ & \multicolumn{3}{|c|}{0.3} \\
\hline$\nu_{p}$ & \multicolumn{3}{|c|}{0.05} \\
\hline$h_{p}(\mathrm{~mm})$ & 10 & 0.01 & \\
\hline$\eta_{p}^{\text {visc }}$ & \multicolumn{2}{|c|}{0.01} \\
\hline
\end{tabular}

Table 3: Characteristics of the plates for the Cavity/Plate/Cavity test case.

\begin{tabular}{|c|c|c|}
\hline & Cavity A & Cavity B \\
\hline$L_{x}(\mathrm{~m})$ & \multicolumn{2}{|c|}{0.9} \\
\hline$L_{y}(\mathrm{~m})$ & \multicolumn{2}{|c|}{0.7} \\
\hline$L_{z}(\mathrm{~m})$ & 1 & 1.2 \\
\hline$\rho_{c}\left(\mathrm{~kg} / \mathrm{m}^{3}\right)$ & \multicolumn{2}{|c|}{1.2} \\
\hline$c_{c}(\mathrm{~m} / \mathrm{s})$ & \multicolumn{2}{|c|}{340} \\
\hline$\eta_{c}^{\text {struc }}$ & \multicolumn{2}{|c|}{0.01} \\
\hline
\end{tabular}

Table 4: Characteristics of the cavities for the Cavity/Plate/Cavity test case. 
[1] R. H. LYON, G. MAIDANIK, Power flow between linearly coupled oscillators, The Journal of the Acoustical Society of America, 34(5) (1962) 623-639.

[2] D. E. NEWLAND, Calculation of power flow between coupled oscillators, Journal of Sound and Vibration, 3(3) (1966) 262-276.

[3] R. H. LYON, R.G. DEJONG, Theory and application of Statistical Energy Analysis, London : Butterworth-Heineman, 1995.

[4] L. JI, B.R. MACE, Statistical energy analysis modelling complex structures as coupled sets of oscillators: Ensemble mean and variance of energy, Journal of Sound and Vibration 317 (2008) 760-780.

[5] V. COTONI, R.S. LANGLEY, M.R.F. KIDNER, Numerical and experimental validation of variance prediction in the statistical energy analysis of built-up systems, Journal of Sound and Vibrations 288 (2005) 701-728.

[6] B.R. MACE, Statistical energy analysis : coupling loss factors, indirect coupling and system modes, Journal of Sound and Vibration 279 (2005) 141-170

[7] A. LE BOT, V. COTONI, Validity diagrams of Statistical Energy Analysis, Journal of Sound and Vibration 329(2) (2010) 221-235.

[8] M.J. CROCKER, A.J. PRICE, Sound transmission using Statistical Energy Analysis, Journal of Sound and Vibration 9 (1969) 469-486.

[9] R.J.M. CRAIK, Non-resonant sound transmission through double walls using Statistical Energy Analysis, Applied Acoustics 64 (2003) 325-341.

[10] K. RENJI, P.S. NAIR, S. NARAYANAN, Non-resonant response using Statistical Energy Analysis, Journal of Sound and Vibration 241(2) (2001) 253-270

[11] L. MAXIT, J.L. GUYADER, Extension of the SEA model to subsystems with non-uniform modal energy distribution, Journal of Sound and Vibration 265(2) (2003) 337-358.

[12] N. TOTARO, C. DODARD, J.L. GUYADER, SEA Coupling Loss Factors of complex vibro-acoustic systems, Journal of Vibration and Acoustics 131(2) (2009).

[13] N. TOTARO, J.L. GUYADER, Extension of the statistical modal energy distribution analysis for estimating energy density in coupled subsystems, Journal of Sound and Vibration 331 (2012) 31143129.

[14] D. KARNOPP, Coupled vibratory-system analysis, using the dual formulation, Journal of the Acoustical Society of America, 40(2) (1966) 380-384.

[15] CDValor, a vibro-acoustic benchmark for simple structures, EA 27, INRS (2006).

[16] C. CACCIOLATI, P. NEPLE, E. GUYADER, J.L. GUYADER, N. TOTARO, Comparison of the vibroacoustic behavior of a rectangular thin plate excited by a diffuse sound field and a turbulent boundary layer, Proceedings of ICSV13, Vienna (Austria), (2006).

\section{List of Figures}

1 Sketch of two oscillators coupled by a gyroscopic coupling . . . . . . . . 12

2 Coupling factor $\alpha_{12}$ as a function of frequency and of gyroscopic coupling coefficient. Top figure represents a cut of this map for $\gamma=500 . \ldots . . .13$

3 Total energies of oscillators 1 (solid black line) and 2 (dashed gray line) when oscillator 1 is excited by a unit force for (a) $\gamma=10$, (c) $\gamma=500$ and (e) $\gamma=1000$ and corresponding transmitted power from oscillator 1 to oscillator 2 for (b) $\gamma=10$, (d) $\gamma=500$ and (f) $\gamma=1000$. Vertical dotted lines represent the positions of eigen-frequencies of uncoupled oscillators.

4 Critical coefficient $\gamma^{\text {crit }}$ as a function of frequency. The dashed line represents $\gamma=500$. The critical coefficient defines the limit between a "thermodynamic" behavior $\left(\alpha_{i j}>0\right)$ and an "anti-thermodynamic" behavior $\left(\alpha_{i j}<0\right) \ldots \ldots \ldots \ldots \ldots \ldots \ldots \ldots \ldots \ldots$ 
5 Power transmitted from oscillator 1 to oscillator 2 when oscillators 1 and 2 are excited by two correlated forces with (a) $\left|F_{1}\right| /\left|F_{2}\right|=10^{1}$, (b) $\left|F_{1}\right| /\left|F_{2}\right|=10^{2}$, (c) $\left|F_{1}\right| /\left|F_{2}\right|=10^{3}$ and (d) $\left|F_{1}\right| /\left|F_{2}\right|=10^{4}$. Black solid line: exact analytical solution, gray dashed line: MODENA approach. Vertical dotted lines represent the positions of eigen-frequencies of uncoupled oscillators. . . . . . . . . . . . . . . .

6 Power transmitted from oscillator 1 to oscillator 2 when oscillators 1 and 2 are excited by two forces with random phase with (a) $\left|F_{1}\right| /\left|F_{2}\right|=10^{1}$, (b) $\left|F_{1}\right| /\left|F_{2}\right|=10^{2}$, (c) $\left|F_{1}\right| /\left|F_{2}\right|=10^{3}$ and (d) $\left|F_{1}\right| /\left|F_{2}\right|=10^{4}$. Black solid line: averaged analytical solution over 2500 realizations with random phase from 0 to $2 \pi$; gray dashed line: MODENA approach with two correlated forces. Vertical dotted lines represent the positions of eigen-frequencies of uncoupled oscillators. The inserted figures show each of 2500 realizations with random phase (in gray) compared to the average response (in black).

7 Coupling between two sets of modes . . . . . . . . . . . . .

8 Sketch of Plate/Cavity coupling test case. The plate is excited by a point

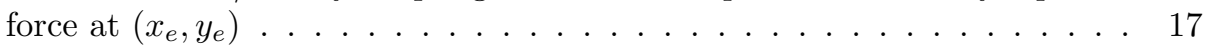

9 Modal energies of modes of (a) the plate and (b) the cavity when the plate is excited by a single point force. Red dashed lines represent the modal energies of the first mode of (a) the plate and (b) the cavity. Green dashed-dotted line represents the modal energy of the $16^{\text {th }}$ mode of the plate. . . . . . . . . . . . . . . . . . .

10 Modal energies of modes of (a) the plate and (b) the cavity when the plate is excited by a rain-on-the-roof excitation (100 uncorrelated point forces of amplitude $1 / 100 \mathrm{~N}$ ). Red dashed lines represent the modal energies of the first mode of (a) the plate and (b) the cavity. Green dashed-dotted line represents the modal energy of the $16^{\text {th }}$ mode of the plate. . . . . . .

11 Total energy of (a) the plate and (b) the cavity as a function of frequency. Plain black line : CVALOR reference calculation; dashed red line : MODENA calculation. The plate is excited by one point force. . . . . . . . . 18

12 Sketch of Cavity/Plate/Cavity coupling test case. The cavity is excited by a monopole at $\left(X_{e}, Y_{e}, Z_{e}\right) \ldots \ldots \ldots \ldots$

13 Energy ratio between cavity A and cavity B when cavity A is excited by one monopole. Black solid line : ACTRAN reference calculation; Dashed red line : MODENA calculation. . . . . . . . . . . . . . . 18

14 Energy ratio between cavity A and cavity B when cavity A is excited by 20 randomly located monopoles. Plain red line : MODENA calculation (Plate 2LD, damping 1\%); plain black line : MODENA calculation (Plate $2 \mathrm{HD}$, damping $5 \%) . \ldots \ldots \ldots \ldots \ldots \ldots \ldots$

\section{List of Tables}

1 Characteristics of the plate for the Plate/Cavity test case . . . . . . 20

2 Characteristics of the cavity for the Plate/Cavity test case . . . . . . . 20

3 Characteristics of the plates for the Cavity/Plate/Cavity test case. . . . . 20

4 Characteristics of the cavities for the Cavity/Plate/Cavity test case. . . . 20 